\title{
Pseudo-almost periodic solutions for a discrete Nicholson's blowflies model with harvesting term
}

\author{
Hui-Sheng Ding* and Meng-Xuan Ji
}

\section{"Correspondence: \\ dinghs@mail.ustc.edu.cn \\ College of Mathematics and \\ Information Science, Jiangxi Normal \\ University, Nanchang, Jiangxi \\ 330022, People's Republic of China}

\begin{abstract}
This paper is concerned with a discrete Nicholson's blowflies model, which involves a linear harvesting term. In the case where the coefficients are pseudo-almost periodic functions, we establish the existence and local exponential stability of pseudo-almost periodic solutions for the addressed Nicholson's blowflies model by using the contraction mapping theorem and a Lyapunov functional. An example is given to illustrate our results.
\end{abstract}

MSC: 34K14

Keywords: pseudo-almost periodic; Nicholson's blowflies model; discrete; difference equations

\section{Introduction and preliminaries}

In 1954, Nicholson [1] and later in 1980, Gurney et al. [2] proposed the following delay differential equation model:

$$
x^{\prime}(t)=-\delta x(t)+p x(t-\tau) e^{-\gamma x(t-\tau)},
$$

where $x(t)$ is the size of the population at time $t, p$ is the maximum per capita daily egg production, $\frac{1}{\gamma}$ is the size at which the population reproduces at its maximum rate, $\delta$ is the per capita daily adult death rate, and $\tau$ is the generation time.

Now, Nicholson's blowflies model and its various analogous equations have attracted more and more attention. There is a wide literature on this topic (see [1-16]). Especially, several authors have made contributions on a discrete Nicholson's blowflies model (see, e.g., $[3-5,15,16]$ and references therein). On the other hand, since the harvest of population species is commonly practiced in fishery, forestry and wildlife management, the study of population dynamics with harvesting has become an important subject. Especially, Berezansky et al. [6] proposed the following Nicholson's blowflies model with linear harvesting term:

$$
x^{\prime}(t)=-\delta x(t)+p x(t-\tau) e^{-\gamma x(t-\tau)}-H(t-\sigma) .
$$

\section{Springer}


Since the work of Berezansky et al. [6], it has attracted much attention of many researchers to study the qualitative properties of the Nicholson's blowflies model with harvesting term. We refer the reader to $[7,9-11,13,14,16]$ and references therein for some of recent works on the Nicholson's blowflies model with harvesting term.

Stimulated by the works mentioned, in this paper, we aim to study the following discrete Nicholson's blowflies model involving a linear harvesting term:

$$
\Delta x(n)=-\alpha(n) x(n)+\beta(n) x(n-\tau(n)) e^{-\gamma(n) x(n-\tau(n))}-H(n) x(n-\sigma(n)),
$$

where $n \in \mathbb{Z}$, and $\alpha, \beta, \gamma, \tau, \sigma, H$ are all pseudo-almost periodic sequences.

Recently, Alzabut et al. [3, 4] investigated the existence and stability of almost periodic solutions for equation (1.2). Yao [16] studied the existence and stability of almost periodic solutions for continuous case of equation (1.2). Chérif [7] and Duan and Huang [9] investigated the existence and stability of pseudo-almost periodic solutions for the continuous case of equation (1.2). However, it seems that there are no results concerning pseudoalmost periodic solutions for discrete equation (1.2), which is the main motivation for this paper.

It is worth noting that the behavior of pseudo-almost periodic functions is more tricky than that of almost periodic functions. In fact, in [7, 9], not all the coefficients are assumed to be pseudo-almost periodic (some coefficients are assumed to be almost periodic). In this paper, we try to study this problem in the case where all coefficients are pseudo-almost periodic functions. As we will see, the pseudo-almost periodicity of $\alpha, \tau, \sigma$ causes some extra difficulties.

Throughout this paper, we denote by $\mathbb{R}$ the set of real numbers, by $\mathbb{R}^{+}$the set of nonnegative real numbers, by $\mathbb{N}$ the set of positive integers, by $\mathbb{Z}$ the set of integers, by $\mathbb{Z}^{+}$ the set of nonnegative integers, and by card $S$ the number of elements in a subset $S$ of $\mathbb{Z}$. Moreover, for every bounded sequence $f: \mathbb{Z} \rightarrow \mathbb{R}$, we denote

$$
f^{+}=\sup _{n \in \mathbb{Z}} f(n), \quad f^{-}=\inf _{n \in \mathbb{Z}} f(n) .
$$

In addition, for some notations related to the initial value problem of equation (1.2), we refer the reader to some earlier references (see, e.g., [3]).

Next, let us recall some basic definitions and results about almost periodic sequences. For more details, we refer the reader to [17-20].

Definition 1.1 A set $E \subset \mathbb{Z}$ is called relatively dense if there exists $l \in \mathbb{N}$ such that

$$
[n, n+l] \cap \mathbb{Z} \cap E \neq \varnothing
$$

for every $n \in \mathbb{Z}$.

Definition 1.2 A function $f: \mathbb{Z} \rightarrow \mathbb{R}$ is called an almost periodic sequence if for every $\varepsilon>0$,

$$
P(\varepsilon, f)=\{\tau \in \mathbb{Z}:|f(n+\tau)-f(n)|<\varepsilon \text { for all } n \in \mathbb{Z}\}
$$

is a relatively dense set in $\mathbb{Z}$. We denote by $A P(\mathbb{Z}, \mathbb{R})$ the set of all such functions. 
Next, we denote by $P A P_{0}(\mathbb{Z}, \mathbb{R})$ the space of all bounded functions $f: \mathbb{Z} \rightarrow \mathbb{R}$ such that

$$
\lim _{n \rightarrow \infty} \frac{1}{2 n} \sum_{k=-n}^{n}|f(k)|=0 .
$$

Lemma $1.3([20])$ Let $f: \mathbb{Z} \rightarrow \mathbb{R}$ be bounded. Then $f \in P A P_{0}(\mathbb{Z}, \mathbb{R})$ if and only iffor every $\varepsilon>0$,

$$
\lim _{n \rightarrow \infty} \frac{\operatorname{card}\{k \in[-n, n] \cap \mathbb{Z}:|f(k)| \geq \varepsilon\}}{2 n}=0 .
$$

Definition 1.4 A function $f: \mathbb{Z} \rightarrow \mathbb{R}$ is called a pseudo-almost periodic sequence if it admits a decomposition $f=g+h$, where $g \in A P(\mathbb{Z}, \mathbb{R})$ and $h \in P A P_{0}(\mathbb{Z}, \mathbb{R})$. We denote by $P A P(\mathbb{Z}, \mathbb{R})$ the set of all such functions.

Lemma $1.5([17-20])$ Let $X \in\{A P(\mathbb{Z}, \mathbb{R}), P A P(\mathbb{Z}, \mathbb{R})\}$. Then:

(a) every $f \in X$ is bounded;

(b) iff, $g \in X$, then $f+g \in X$ and $f \cdot g \in X$;

(c) $X$ is a Banach space equipped with the supremum norm;

(d) $f \in X$ implies that $f(\cdot+s) \in X$ for every $s \in \mathbb{R}$;

(d) $f \in X$ implies that $F \circ f \in X$ for every continuous function $F: \mathbb{R} \rightarrow \mathbb{R}$.

Now, let us recall some basic results about the linear difference system

$$
x(n+1)=A(n) x(n), \quad n \in \mathbb{Z},
$$

where for every $n \in \mathbb{Z}, x(n) \in \mathbb{R}^{q}$ and $A(n)$ is an invertible $q \times q$ matrix. Denote

$$
\Phi(n, m)= \begin{cases}A(n-1) \cdots A(m), & n>m, \\ I, & n=m, \\ A^{-1}(n) \cdots A^{-1}(m), & n<m .\end{cases}
$$

Definition 1.6 ([19]) We say that the linear difference system (1.3) has an exponential dichotomy on $\mathbb{Z}$ if there are positive constants $K, \lambda$ and a family of projections $P(n)$ such that

$$
P(n+1) A(n)=A(n) P(n), \quad n \in \mathbb{Z},
$$

and

$$
|\Phi(n, m) P(m)| \leq K e^{-\lambda(n-m)}, \quad n \geq m, \quad|\Phi(n, m)(I-P(m))| \leq K e^{-\lambda(m-n)}, \quad m>n .
$$

Lemma 1.7 ([19]) If the linear difference system (1.3) has an exponential dichotomy on $\mathbb{Z}$ and $f: \mathbb{Z} \rightarrow \mathbb{R}$ is bounded, then the inhomogeneous system

$$
x(n+1)=A(n) x(n)+f(n), \quad n \in \mathbb{Z},
$$


has a unique bounded solution given by

$$
x(n)=\sum_{m=-\infty}^{n-1} \Phi(n, m+1) P(m+1) f(m)-\sum_{m=n}^{+\infty} \Phi(n, m+1)(I-P(m+1)) f(m), \quad n \in \mathbb{Z} .
$$

\section{Main results}

Before presenting our main results, let us first establish some lemmas. For every subset $\Omega \subset \mathbb{R}$, we denote by $P A P(\mathbb{Z}, \Omega)$ the set of all functions $f \in P A P(\mathbb{Z}, \Omega)$ with $f(\mathbb{Z}) \subset \Omega$. We also use the notations $A P(\mathbb{Z}, \Omega)$ and $P A P_{0}(\mathbb{Z}, \Omega)$, which are similar to $P A P(\mathbb{Z}, \Omega)$.

Lemma 2.1 Let $f \in P A P(\mathbb{Z}, \mathbb{Z})$. Then, there exist periodic functions $f_{1}: \mathbb{Z} \rightarrow \mathbb{Z}$ and $f_{2} \in$ $P A P_{0}(\mathbb{Z}, \mathbb{Z})$ such that $f=f_{1}+f_{2}$.

Proof Let $f=f_{1}+f_{2}$, where $f_{1} \in A P(\mathbb{Z}, \mathbb{R})$ and $f_{2} \in P A P_{0}(\mathbb{Z}, \mathbb{R})$. By Lemma 2.7 in [20] we have

$$
\left\{f_{1}(n): n \in \mathbb{Z}\right\} \subset \overline{\{f(n): n \in \mathbb{Z}\}} \subset \mathbb{Z},
$$

and we conclude that $f_{1} \in A P(\mathbb{Z}, \mathbb{Z})$ and $f_{2} \in P A P_{0}(\mathbb{Z}, \mathbb{Z})$. Moreover, since $f_{1} \in A P(\mathbb{Z}, \mathbb{Z})$, there exists $p>0$ such that

$$
\sup _{n \in \mathbb{Z}}\left|f_{1}(n+p)-f_{1}(n)\right|<\frac{1}{2}
$$

which means that $f_{1}(n+p)=f_{1}(n)$ for all $n \in \mathbb{Z}$, that is, $f_{1}$ is a periodic function.

Example 2.2 Let

$$
\sigma_{1}(n)=\left\{\begin{array}{ll}
1, & n=2 k+1, k \in \mathbb{Z}, \\
2, & n=2 k, k \in \mathbb{Z},
\end{array} \quad \text { and } \quad \sigma_{2}(n)= \begin{cases}1, & n=2^{k}, k=1,2, \ldots \\
0, & \text { otherwise }\end{cases}\right.
$$

It is not difficult to verify that $\sigma=\sigma_{1}+\sigma_{2} \in P A P(\mathbb{Z}, \mathbb{Z})$.

Lemma 2.3 Let $\phi \in P A P(\mathbb{Z}, \mathbb{R})$ and $\tau \in P A P(\mathbb{Z}, \mathbb{Z})$. Then, $\phi(\cdot-\tau(\cdot)) \in P A P(\mathbb{Z}, \mathbb{R})$.

Proof Let $\phi=\phi_{1}+\phi_{2}$ and $\tau=\tau_{1}+\tau_{2}$, where $\phi_{1} \in A P(\mathbb{Z}, \mathbb{R}), \phi_{2} \in P A P_{0}(\mathbb{Z}, \mathbb{R}), \tau_{1} \in A P(\mathbb{Z}, \mathbb{Z})$, and $\tau_{2} \in P A P_{0}(\mathbb{Z}, \mathbb{Z})$.

For all $n \in \mathbb{Z}$, we have

$$
\begin{aligned}
\phi(n-\tau(n)) & =\phi_{1}(n-\tau(n))+\phi_{2}(n-\tau(n)) \\
& =\phi_{1}\left(n-\tau_{1}(n)\right)+\phi_{1}(n-\tau(n))-\phi_{1}\left(n-\tau_{1}(n)\right)+\phi_{2}(n-\tau(n)) \\
& =I_{1}(n)+I_{2}(n)+I_{3}(n),
\end{aligned}
$$

where $I_{1}(n)=\phi_{1}\left(n-\tau_{1}(n)\right), I_{2}(n)=\phi_{1}(n-\tau(n))-\phi_{1}\left(n-\tau_{1}(n)\right), I_{3}(n)=\phi_{2}(n-\tau(n))$.

For every $\varepsilon \in(0,1)$, noting that $\tau_{1} \in A P(\mathbb{Z}, \mathbb{Z})$, we have

$$
\tau_{1}(n+p)=\tau_{1}(n), \quad n \in \mathbb{Z}, p \in P\left(\varepsilon, \tau_{1}\right),
$$


which yields that, for all $n \in \mathbb{Z}$ and $p \in P\left(\varepsilon, \tau_{1}\right) \cap P\left(\varepsilon, \phi_{1}\right)$,

$$
\begin{aligned}
\left|I_{1}(n+p)-I_{1}(n)\right| & =\left|\phi_{1}\left(n+p-\tau_{1}(n+p)\right)-\phi_{1}\left(n-\tau_{1}(n)\right)\right| \\
& =\left|\phi_{1}\left(n+p-\tau_{1}(n)\right)-\phi_{1}\left(n-\tau_{1}(n)\right)\right|<\varepsilon .
\end{aligned}
$$

Recalling that $P\left(\varepsilon, \tau_{1}\right) \cap P\left(\varepsilon, \phi_{1}\right)$ is still relatively dense in $\mathbb{Z}$, we conclude that $I_{1} \in A P(\mathbb{Z}, \mathbb{R})$.

We claim that, for all $\varepsilon \in(0,1)$ and $n \in \mathbb{N}$,

$$
\left\{k \in[-n, n] \cap \mathbb{Z}:\left|I_{2}(k)\right| \geq \varepsilon\right\} \subset\left\{k \in[-n, n] \cap \mathbb{Z}:\left|\tau_{2}(k)\right| \geq \varepsilon\right\} .
$$

In fact, if $k \notin\left\{k \in[-n, n] \cap \mathbb{Z}:\left|\tau_{2}(k)\right| \geq \varepsilon\right\}$, then $\left|\tau_{2}(k)\right|<\varepsilon<1$, and thus $\tau_{2}(k)=0$ since $\tau_{2}$ is integer valued. So $I_{2}(k)=0$, that is, $k \notin\left\{k \in[-n, n] \cap \mathbb{Z}:\left|I_{2}(k)\right| \geq \varepsilon\right\}$. Since $\tau_{2} \in P A P_{0}(\mathbb{Z}, \mathbb{Z})$, for the above $\varepsilon \in(0,1)$, it follows from Lemma 1.3 that

$$
\lim _{n \rightarrow \infty} \frac{\operatorname{card}\left\{k \in[-n, n] \cap \mathbb{Z}:\left|\tau_{2}(k)\right| \geq \varepsilon\right\}}{2 n}=0 .
$$

Then, by (2.1) we get

$$
\lim _{n \rightarrow \infty} \frac{\operatorname{card}\left\{k \in[-n, n] \cap \mathbb{Z}:\left|I_{2}(k)\right| \geq \varepsilon\right\}}{2 n}=0 .
$$

Again by Lemma 1.3, we conclude that $I_{2} \in P A P_{0}(\mathbb{Z}, \mathbb{R})$.

Let $K=\sup _{n \in \mathbb{Z}}|\tau(n)|$. Then $K \in \mathbb{Z}^{+}$since $\tau \in P A P(\mathbb{Z}, \mathbb{Z})$. Noting that $\phi_{2} \in P A P_{0}(\mathbb{Z}, \mathbb{R})$, we have

$$
\begin{aligned}
\frac{1}{2 n} \sum_{k=-n}^{n}\left|I_{3}(k)\right| & =\frac{1}{2 n} \sum_{k=-n}^{n}\left|\phi_{2}(k-\tau(k))\right| \\
& \leq \frac{1}{2 n} \sum_{k=-n}^{n} \sum_{m=-K}^{K}\left|\phi_{2}(k-m)\right| \\
& =\sum_{m=-K}^{K}\left(\frac{1}{2 n} \sum_{k=-n}^{n}\left|\phi_{2}(k-m)\right|\right) .
\end{aligned}
$$

Combining this with the fact that $P A P_{0}(\mathbb{Z}, \mathbb{R})$ is translation invariant, we get

$$
\lim _{n \rightarrow \infty} \frac{1}{2 n} \sum_{k=-n}^{n}\left|I_{3}(k)\right|=0
$$

Thus, $I_{3} \in P A P_{0}(\mathbb{Z}, \mathbb{R})$.

It follows from the above proof that $\phi(\cdot-\tau(\cdot)) \in P A P(\mathbb{Z}, \mathbb{R})$.

Lemma 2.4 Let $\theta, f \in P A P(\mathbb{Z}, \mathbb{R})$ with $\|\theta\|<1$, and

$$
F(n)=\sum_{m=-\infty}^{n-1}\left[\prod_{i=m+1}^{n-1} \theta(i)\right] f(m), \quad n \in \mathbb{Z},
$$

where we denote $\prod_{i=n}^{n-1} \theta(i)=1$ for simplicity. Then $F \in P A P(\mathbb{Z}, \mathbb{R})$. 
Proof It is easy to see that

$$
F(n)=\sum_{k=1}^{+\infty}\left[\prod_{i=n-k+1}^{n-1} \theta(i)\right] f(n-k), \quad n \in \mathbb{Z} .
$$

For every $l \in \mathbb{N}$, we denote

$$
F_{l}(n)=\sum_{k=1}^{l}\left[\prod_{i=n-k+1}^{n-1} \theta(i)\right] f(n-k), \quad n \in \mathbb{Z} .
$$

It follows from Lemma 1.5 that $F_{l} \in P A P(\mathbb{Z}, \mathbb{R})$ for every $l \in \mathbb{N}$. On the other hand, we have

$$
\begin{aligned}
\left\|F-F_{l}\right\| & =\sup _{n \in \mathbb{Z}}\left|\sum_{k=l+1}^{+\infty}\left[\prod_{i=n-k+1}^{n-1} \theta(i)\right] f(n-k)\right| \\
& \leq\|f\| \cdot \sum_{k=l+1}^{+\infty}\|\theta\|^{k-1} \\
& =\|f\| \cdot \frac{\|\theta\|^{l}}{1-\|\theta\|} \rightarrow 0, \quad l \rightarrow+\infty .
\end{aligned}
$$

Since $P A P(\mathbb{Z}, \mathbb{R})$ is a Banach space, we conclude that $F \in P A P(\mathbb{Z}, \mathbb{R})$.

For the next existence theorem, we will use the following assumptions:

(A0) $\alpha \in P A P(\mathbb{Z}, \mathbb{R})$ with $0<\alpha^{-} \leq \alpha^{+}<1, \beta, \gamma, H \in P A P\left(\mathbb{Z}, \mathbb{R}^{+}\right)$with $\beta^{-}, \gamma^{-}>0$, and $\tau, \sigma \in P A P\left(\mathbb{Z}, \mathbb{Z}^{+}\right)$.

(A1) $\Gamma_{1}>\Gamma_{2} \geq \frac{1}{\gamma^{+}}$, where

$$
\frac{\beta^{+}}{\alpha^{-} \gamma^{-} e}=\Gamma_{1}, \quad \frac{\Gamma_{1}\left(\beta^{-} e^{-\gamma^{+} \Gamma_{1}}-H^{+}\right)}{\alpha^{+}}=\Gamma_{2} .
$$

(A2) $H^{+}+\beta^{+} \max \left\{\frac{|1-k|}{e^{k}}, \frac{1}{e^{2}}\right\}<\alpha^{-}$, where $k=\Gamma_{2} \gamma^{-}$.

Theorem 2.5 Under assumptions (A0)-(A2), there exists a unique pseudo-almost periodic solution of equation (1.2) in

$$
\Omega=\left\{\varphi \in P A P(\mathbb{Z}, \mathbb{R}): \Gamma_{2} \leq \varphi(n) \leq \Gamma_{1}, \forall n \in \mathbb{Z}\right\}
$$

Proof Taking $\varphi \in \Omega$, consider the following difference equation:

$$
\Delta x(n)=-\alpha(n) x(n)+\beta(n) \varphi(n-\tau(n)) e^{-\gamma(n) \varphi(n-\tau(n))}-H(n) \varphi(n-\sigma(n)),
$$

that is,

$$
x(n+1)=(1-\alpha(n)) x(n)+\beta(n) \varphi(n-\tau(n)) e^{-\gamma(n) \varphi(n-\tau(n))}-H(n) \varphi(n-\sigma(n)) .
$$

By Lemma 2.3 we have

$$
\varphi(\cdot-\tau(\cdot)), \varphi(\cdot-\sigma(\cdot)) \in P A P(\mathbb{Z}, \mathbb{R})
$$


Then, by Lemma 1.5 we conclude that

$$
\beta(\cdot) \varphi(\cdot-\tau(\cdot)) e^{-\gamma(\cdot) \varphi(\cdot-\tau(\cdot))}-H(\cdot) \varphi(\cdot-\sigma(\cdot)) \in \operatorname{PAP}(\mathbb{Z}, \mathbb{R}) .
$$

Noting that $0<\alpha^{-} \leq \alpha^{+}<1, x(n+1)=(1-\alpha(n)) x(n)$ admits an exponential dichotomy on $\mathbb{Z}$ with $P(n) \equiv I$. Then, by Lemma 1.7 we deduce that equation (2.2) has a unique bounded solution given by

$$
x^{\varphi}(n)=\sum_{m=-\infty}^{n-1}\left[\prod_{i=m+1}^{n-1}(1-\alpha(i))\right] \cdot\left(\beta(m) \varphi(m-\tau(m)) e^{-\gamma(m) \varphi(m-\tau(m))}-H(m) \varphi(m-\sigma(m))\right) .
$$

Now, define a mapping on $\Omega$ by

$$
(T \varphi)(n)=x^{\varphi}(n), \quad n \in \mathbb{Z}, \varphi \in \Omega .
$$

It follows from Lemma 2.4 that $T \varphi \in \operatorname{PAP}(\mathbb{Z}, \mathbb{R})$ for every $\varphi \in \Omega$. Next, let us show that $T(\Omega) \subset \Omega$.

Since

$$
\sup _{x \geq 0} x e^{-\gamma^{-} x}=\frac{1}{\gamma^{-} e}
$$

for every $\varphi \in \Omega$ and $n \in \mathbb{Z}$, we have

$$
\begin{aligned}
(T \varphi)(n)= & \sum_{m=-\infty}^{n-1}\left[\prod_{i=m+1}^{n-1}(1-\alpha(i))\right] \\
& \cdot\left(\beta(m) \varphi(m-\tau(m)) e^{-\gamma(m) \varphi(m-\tau(m))}-H(m) \varphi(m-\sigma(m))\right) \\
\leq & \sum_{m=-\infty}^{n-1}\left[\prod_{i=m+1}^{n-1}(1-\alpha(i))\right] \cdot \beta(m) \varphi(m-\tau(m)) e^{-\gamma^{-} \varphi(m-\tau(m))} \\
\leq & \sum_{m=-\infty}^{n-1}\left[\prod_{i=m+1}^{n-1}\left(1-\alpha^{-}\right)\right] \cdot \frac{\beta^{+}}{\gamma^{-} e} \\
= & \frac{\beta^{+}}{\alpha^{-} \gamma^{-} e}=\Gamma_{1} .
\end{aligned}
$$

On the other hand, by (A1) we know that $\Gamma_{2} \geq \frac{1}{\gamma^{+}}$, which yields

$$
\inf _{\Gamma_{2} \leq x \leq \Gamma_{1}} x e^{-\gamma^{+} x}=\Gamma_{1} e^{-\gamma^{+} \Gamma_{1}} .
$$

Thus, we have

$$
\begin{aligned}
(T \varphi)(n)= & \sum_{m=-\infty}^{n-1}\left[\prod_{i=m+1}^{n-1}(1-\alpha(i))\right] \\
& \cdot\left(\beta(m) \varphi(m-\tau(m)) e^{-\gamma(m) \varphi(m-\tau(m))}-H(m) \varphi(m-\sigma(m))\right) \\
\geq & \sum_{m=-\infty}^{n-1}\left[\prod_{i=m+1}^{n-1}\left(1-\alpha^{+}\right)\right] \cdot\left(\beta^{-} \varphi(m-\tau(m)) e^{-\gamma^{+} \varphi(m-\tau(m))}-H^{+} \Gamma_{1}\right)
\end{aligned}
$$




$$
\begin{aligned}
& \geq \sum_{m=-\infty}^{n-1}\left[\prod_{i=m+1}^{n-1}\left(1-\alpha^{+}\right)\right] \cdot\left(\beta^{-} \Gamma_{1} e^{-\gamma^{+} \Gamma_{1}}-H^{+} \Gamma_{1}\right) \\
& =\frac{\Gamma_{1}\left(\beta^{-} e^{-\gamma^{+} \Gamma_{1}}-H^{+}\right)}{\alpha^{+}}=\Gamma_{2}
\end{aligned}
$$

for every $\varphi \in \Omega$ and $n \in \mathbb{Z}$.

We have shown that $T$ is a self-mapping from $\Omega$ to $\Omega$. It is obvious that $\Omega$ is a closed subset in $P A P(\mathbb{Z}, \mathbb{R})$. Next, let us show that $T$ is a contraction. Noting that

$$
\left|x e^{-x}-y e^{-y}\right| \leq \max \left\{\frac{|1-k|}{e^{k}}, \frac{1}{e^{2}}\right\}|x-y|, \quad x, y \geq k
$$

for all $\varphi, \psi \in \Omega$, we have

$$
\begin{aligned}
& \|T(\varphi)-T(\psi)\| \\
& =\sup _{n \in \mathbb{Z}} \mid \sum_{m=-\infty}^{n-1}\left[\prod_{i=m+1}^{n-1}(1-\alpha(i))\right] \cdot\{H(m) \psi(m-\sigma(m))-H(m) \varphi(m-\sigma(m)) \\
& \left.\quad+\beta(m) \varphi(m-\tau(m)) e^{-\gamma(m) \varphi(m-\tau(m))}-\beta(m) \psi(m-\tau(m)) e^{-\gamma(m) \psi(m-\tau(m))}\right\} \mid \\
& \leq \sup _{n \in \mathbb{Z}} \sum_{m=-\infty}^{n-1}\left[\prod_{i=m+1}^{n-1}(1-\alpha(i))\right] \cdot\left\{H^{+}\|\varphi-\psi\|+\beta^{+} \max \left\{\frac{|1-k|}{e^{k}}, \frac{1}{e^{2}}\right\}\|\varphi-\psi\|\right\} \\
& \leq \frac{H^{+}+\beta^{+} \max \left\{\frac{|1-k|}{e^{k}}, \frac{1}{e^{2}}\right\}}{\alpha^{-}}\|\varphi-\psi\|,
\end{aligned}
$$

which implies by (A2) that the mapping $T$ is a contraction. Therefore, $T$ has a unique fixed point in $\Omega$, which means that equation (1.2) has a unique pseudo-almost periodic solution in $\Omega$.

Next, let us discuss locally exponential stability of the pseudo-almost periodic solution of equation (1.2).

Theorem 2.6 Suppose that (A0)-(A2) and

$$
H^{+}+\beta^{+} \max \left\{\frac{|1-k|}{e^{k}}, \frac{1}{e^{2}}\right\}<\frac{1-\alpha^{+}}{1-\alpha^{-}} \cdot \alpha^{-}
$$

are satisfied. Let $x^{*}$ be the unique pseudo-almost periodic solution of equation (1.2) in $\Omega$, and $x$ be an arbitrary solution of equation (1.2) with $\inf _{n \geq-N} x(t) \geq \Gamma_{2}$. Then, there exists a constant $\lambda>0$ such that

$$
\left|x(n)-x^{*}(n)\right| \leq M e^{-\lambda n}, \quad n \geq-N,
$$

where $N=\max \left\{\tau^{+}, \sigma^{+}\right\}$and $M=\max _{-N \leq n \leq 0}\left|x(n)-x^{*}(n)\right|$.

Proof Let $y(n)=x(n)-x^{*}(n), n \geq-N$. Then

$$
\begin{aligned}
\Delta y(n)= & -\alpha(n) y(n)+\beta(n)\left[x(n-\tau(n)) e^{-\gamma(n) x(n-\tau(n))}-x^{*}(n-\tau(n)) e^{-\gamma(n) x^{*}(n-\tau(n))}\right] \\
& -H(n) y(n-\sigma(n)),
\end{aligned}
$$


that is,

$$
\begin{aligned}
\Delta y(n)= & -\frac{\alpha(n)}{1-\alpha(n)} y(n+1) \\
& +\frac{\beta(n)}{1-\alpha(n)}\left[x(n-\tau(n)) e^{-\gamma(n) x(n-\tau(n))}-x^{*}(n-\tau(n)) e^{-\gamma(n) x^{*}(n-\tau(n))}\right] \\
& -\frac{H(n)}{1-\alpha(n)} y(n-\sigma(n)) .
\end{aligned}
$$

Letting $\tilde{k}=\max \left\{\frac{|1-k|}{e^{k}}, \frac{1}{e^{2}}\right\}$, by (A3) there exists $\lambda>0$ such that

$$
\frac{H^{+}}{1-\alpha^{+}} e^{\lambda(N+1)}+e^{\lambda}-1+\frac{\beta^{+}}{1-\alpha^{+}} \tilde{k} e^{\lambda(N+1)}<\frac{\alpha^{-}}{1-\alpha^{-}} .
$$

Consider the discrete Lyapunov functional

$$
V(n)=|y(n)| e^{\lambda n}, \quad n \geq-N .
$$

It is easy to see that $V(n) \leq M$ for all $n \in[-N, 0] \cap \mathbb{Z}$. We claim that $V(n) \leq M$ for all $n \geq-N$. In fact, if this were not true, then

$$
\{n>0: V(n)>M\} \neq \emptyset .
$$

Set

$$
n_{0}=\min \{n>0: V(n)>M\}-1 .
$$

Then $n_{0} \geq 0$ and

$$
V\left(n_{0}+1\right)>M, \quad V(n) \leq M, \quad n \in\left[-N, n_{0}\right] \cap \mathbb{Z} .
$$

Combining this with (2.4), we get that

$$
\begin{aligned}
0< & V\left(n_{0}+1\right)-V\left(n_{0}\right) \\
= & \Delta V\left(n_{0}\right)=\Delta\left(\left|y\left(n_{0}\right)\right| e^{\lambda n_{0}}\right) \\
= & \Delta\left|y\left(n_{0}\right)\right| e^{\lambda\left(n_{0}+1\right)}+\left|y\left(n_{0}\right)\right| \Delta e^{\lambda n_{0}} \\
\leq & -\frac{\alpha\left(n_{0}\right)}{1-\alpha\left(n_{0}\right)}\left|y\left(n_{0}+1\right)\right| e^{\lambda\left(n_{0}+1\right)}+\frac{H\left(n_{0}\right)}{1-\alpha\left(n_{0}\right)}\left|y\left(n_{0}-\sigma\left(n_{0}\right)\right)\right| e^{\lambda\left(n_{0}+1\right)} \\
& +\left|y\left(n_{0}\right)\right|\left(e^{\lambda\left(n_{0}+1\right)}-e^{\lambda n_{0}}\right)+\frac{\beta\left(n_{0}\right)}{1-\alpha\left(n_{0}\right)} \mid x\left(n_{0}-\tau\left(n_{0}\right)\right) e^{-\gamma\left(n_{0}\right) x\left(n_{0}-\tau\left(n_{0}\right)\right)} \\
& -x^{*}\left(n_{0}-\tau\left(n_{0}\right)\right) e^{-\gamma\left(n_{0}\right) x^{*}\left(n_{0}-\tau\left(n_{0}\right)\right)} \mid e^{\lambda\left(n_{0}+1\right)} \\
\leq & -\frac{\alpha\left(n_{0}\right)}{1-\alpha\left(n_{0}\right)} V\left(n_{0}+1\right)+\frac{H^{+}}{1-\alpha^{+}} V\left(n_{0}-\sigma\left(n_{0}\right)\right) e^{\lambda \sigma\left(n_{0}\right)} e^{\lambda} \\
& +V\left(n_{0}\right)\left(e^{\lambda}-1\right)+\frac{\beta^{+}}{1-\alpha^{+}} \tilde{k} y\left(n_{0}-\tau\left(n_{0}\right)\right) e^{\lambda\left(n_{0}+1\right)}
\end{aligned}
$$




$$
\begin{aligned}
& \leq-\frac{\alpha^{-}}{1-\alpha^{-}} M+\frac{H^{+}}{1-\alpha^{+}} M e^{\lambda(N+1)}+M\left(e^{\lambda}-1\right)+\frac{\beta^{+}}{1-\alpha^{+}} \tilde{k} V\left(n_{0}-\tau\left(n_{0}\right)\right) e^{\lambda \tau\left(n_{0}\right)} e^{\lambda} \\
& \leq-\frac{\alpha^{-}}{1-\alpha^{-}} M+\frac{H^{+}}{1-\alpha^{+}} M e^{\lambda(N+1)}+M\left(e^{\lambda}-1\right)+\frac{\beta^{+}}{1-\alpha^{+}} \tilde{k} M e^{\lambda(N+1)} \\
& =M\left(-\frac{\alpha^{-}}{1-\alpha^{-}}+\frac{H^{+}}{1-\alpha^{+}} e^{\lambda(N+1)}+e^{\lambda}-1+\frac{\beta^{+}}{1-\alpha^{+}} \tilde{k} e^{\lambda(N+1)}\right) .
\end{aligned}
$$

Thus, we have

$$
\frac{H^{+}}{1-\alpha^{+}} e^{\lambda(N+1)}+e^{\lambda}-1+\frac{\beta^{+}}{1-\alpha^{+}} \tilde{k} e^{\lambda(N+1)}>\frac{\alpha^{-}}{1-\alpha^{-}},
$$

which contradicts with (2.5). So $V(n) \leq M$ for all $n \geq-N$, that is,

$$
\left|x(n)-x^{*}(n)\right| \leq M e^{-\lambda n}, \quad n \geq-N .
$$

At last, we give an example to show that (A0)-(A3) can be satisfied. For convenience of calculation, the following example does not aim at generality.

Example 2.7 Let $\tau=\sigma$ be as in Example 2.2, $\beta(n) \equiv e, \gamma(n) \equiv 1$, and

$$
\alpha(n)=\left\{\begin{array}{ll}
\frac{5}{8}, & n=2^{k}, k=1,2, \ldots, \\
\frac{1}{2}, & \text { otherwise }
\end{array} \quad \text { and } \quad H(n)=\frac{\cos ^{2} n+\cos ^{2} \sqrt{2} n+e^{-n^{2}}}{600} .\right.
$$

By a direct calculation we get

$$
\alpha^{-}=\frac{1}{2}, \quad \alpha^{+}=\frac{5}{8}, \quad \beta^{+}=\beta^{-}=e, \quad \gamma^{+}=\gamma^{-}=1, \quad H^{+}=\frac{1}{200} .
$$

It is easy to see that (A0) holds. Moreover,

$$
\Gamma_{1}=\frac{\beta^{+}}{\alpha^{-} \gamma^{-} e}=2, \quad \Gamma_{2}=\frac{\Gamma_{1}\left(\beta^{-} e^{-\gamma^{+} \Gamma_{1}}-H^{+}\right)}{\alpha^{+}}=\frac{16}{5}\left(\frac{1}{e}-\frac{1}{200}\right) .
$$

It is easy to see that $\Gamma_{1}>\Gamma_{2} \geq \frac{1}{\gamma^{+}}$, that is, (A1) holds. Moreover, we have

$$
H^{+}+\beta^{+} \max \left\{\frac{|1-k|}{e^{k}}, \frac{1}{e^{2}}\right\}=\frac{1}{200}+\frac{1}{e}<\frac{3}{8}=\frac{1-\alpha^{+}}{1-\alpha^{-}} \cdot \alpha^{-}<\alpha^{-},
$$

which means that (A2) and (A3) hold. This means that all the assumption of Theorem 2.5 and Theorem 2.6 are satisfied. Thus, equation (1.2) has a unique pseudo-almost periodic solution $x^{*}$ in $\Omega$, and $x^{*}$ is locally exponentially stable.

\section{Competing interests}

The authors declare that they have no competing interests.

\section{Authors' contributions}

Both authors contributed equally in this work. Both authors read and approved the final manuscript.

\section{Acknowledgements}

The work was partially supported by NSFC (11461034), the Program for Cultivating Young Scientist of Jiangxi Province (20133BCB23009), the NSF of Jiangxi Province (20143ACB21001), and the Foundation of Jiangxi Provincial Education Department (GJJ150342). 


\section{References}

1. Nicholson, AJ: An outline of the dynamics of animal populations. Aust. J. Zool. 2, 9-65 (1954)

2. Gurney, WS, Blythe, SP, Nisbet, RM: Nicholson's blowflies revisited. Nature 287, $17-21$ (1980)

3. Alzabut, JO, Bolat, Y, Abdeljawad, T: Almost periodic dynamics of a discrete Nicholson's blowflies model involving a linear harvesting term. Adv. Differ. Equ. 2012, 158 (2012)

4. Alzabut, JO: Existence and exponential convergence of almost periodic solutions for a discrete Nicholson's blowflies model with a nonlinear harvesting term. Math. Sci. Lett. 2, 201-207 (2013)

5. Alzabut, J, Obaidat, S, Yao, Z: Exponential extinction of discrete Nicholson's blowflies systems with patch structure and mortality terms. J. Math. Comput. Sci. 16, 298-307 (2016)

6. Berezansky, L, Braverman, E, Idels, L: Nicholson's blowflies differential equations revisited: main results and open problems. Appl. Math. Model. 34, 1405-1417 (2010)

7. Chérif, F: Pseudo almost periodic solution of Nicholson's blowflies model with mixed delays. Appl. Math. Model. 39 5152-5163 (2015)

8. Ding, HS, Dix, JG: Multiple periodic solutions for discrete Nicholson's blowflies type system. Abstr. Appl. Anal. 2014, Article ID 659152 (2014)

9. Duan, L, Huang, LH: Pseudo almost periodic dynamics of delay Nicholson's blowflies model with a linear harvesting term. Math. Methods Appl. Sci. 38, 1178-1189 (2015)

10. Liu, BW: Global dynamic behaviors for a delayed Nicholson's blowflies model with a linear harvesting term. Electron. J. Qual. Theory Differ. Equ. 2013, 45 (2013)

11. Padhi, S, Pati, S, Kumar, R: Positive periodic solutions of Nicholson's blowflies model with harvesting. Panam. Math. J. 24, 15-26 (2014)

12. Saker, SH: Oscillation of continuous and discrete diffusive delay Nicholson's blowflies models. Appl. Math. Comput. 167, 179-197 (2005)

13. Tunç, C, Liu, BW: Global stability of pseudo almost periodic solutions for a Nicholson's blowflies model with a harvesting term. Vietnam J. Math. 44, 485-494 (2016)

14. Wang, L: Almost periodic solution for Nicholson's blowflies model with patch structure and linear harvesting terms. Appl. Math. Model. 37, 2153-2165 (2013)

15. So, JW-H, Yu, JS: On the stability and uniform persistence of a discrete model of Nicholson's blowflies. J. Math. Anal. Appl. 193, 233-244 (1995)

16. Yao, ZJ: Existence and exponential convergence of almost periodic positive solution for Nicholson's blowflies discrete model with linear harvesting term. Math. Methods Appl. Sci. 37, 2354-2362 (2014)

17. Corduneanu, C: Almost Periodic Functions, 2nd edn. Chelsea, New York (1989)

18. Diagana, T: Pseudo Almost Periodic Functions in Banach Spaces. Nova Science Publishers, New York (2007)

19. Zhang, C: Almost Periodic Type Functions and Ergodicity. Science Press, Beijing; Kluwer Academic, Dordrecht (2003)

20. Ding, HS, Fu, JD, N'Guérékata, GM: Positive almost periodic type solutions to a class of nonlinear difference equations. Electron. J. Qual. Theory Differ. Equ. 2011, 25 (2011)

\section{Submit your manuscript to a SpringerOpen ${ }^{\ominus}$ journal and benefit from:}

- Convenient online submission

- Rigorous peer review

- Immediate publication on acceptance

Open access: articles freely available online

- High visibility within the field

- Retaining the copyright to your article 